\title{
Integrating Mental Health into General Health Care: Lessons From HIV
}

\author{
JA Joska', KR Sorsdahl² \\ 1Department of Psychiatry and Mental Health, Division of Neuropsychiatry, University of Cape Town, Cape Town, South Africa \\ 2Department of Psychiatry and Mental Health, Brain and Behaviour Initiative, University of Cape Town, Cape Town, South Africa
}

\begin{abstract}
Mental disorders are highly prevalent across all health settings. Where they are co-morbid with other chronic physical disorders, a complex bidirectional relationship exists between them. While mental disorders may result in an increase in adverse healthrelated outcomes, they are amenable to cost-effective treatments. In resource-limited settings, many barriers to the detection and treatment of mental disorders exist. One approach to the effective targeting of the available resources is to utilize a "risk-flag" approach, wherein individuals at-risk of treatment failure are identified and routed into more intensive mental health screening and intervention. This paper discusses how lessons from HIV services may inform how to improve mental health care and integration in HIV settings, as well as in other chronic diseases.
\end{abstract}

Keywords: Mental disorders; Mental Health Services; Medication Adherence; South Africa

Received: 08-06-2012

Accepted: 19-07-2012

doi: http://dx.doi.org/10.4314/ajpsy.v15i6.52

This article is based on a paper presented at the National Mental Health Summit convened by the Department of Health, Republic of South Africa from 12-13 April 2012.

\section{Introduction}

The World Health Organisation has strongly espoused the notion that "there is no health without mental health". 1 Underpinning this assertion is the finding that at least $25 \%$ of patients attending or presenting to primary health care services have a mental or neurologic disorder. ${ }^{2}$ Furthermore, there is strong evidence suggesting a complex bidirectional relationship between mental and chronic physical disorders. In the first instance, chronic communicable and noncommunicable diseases are associated with higher than expected rates of mental disorders (see for example Ciesla and Roberts ${ }^{3}$ ). Conversely, individuals with mental disorders may be at risk of acquiring chronic diseases: those with severe mental illnesses, may be at risk of HIV infection. ${ }^{4}$ These interrelated processes suggest high rates of co-morbidity.

There is little dispute that untreated mental disorders affect

\section{Correspondence}

Prof. JA Joska

Division of Neuropsychiatry, Department of Psychiatry and Mental Health,

University of Cape Town, Groote Schuur Hospital, Anzio Road,

Observatory, 7925, Cape Town, South Africa

email: John.Joska@uct.ac.za many health- and general outcomes in people living with chronic diseases. In the HIV literature, studies have demonstrated delayed initiation of treatment, impaired adherence, more rapid progression of disease, and increased rates of mortality. ${ }^{5 ; 6 ;}$ Recently, similar findings have been reported amongst other chronic non-communicable diseases. ${ }^{8}$ The impact of the increased burden of diseases is substantial in health, societal and individual costs.

\section{"Health" and its proxies}

The goal of all medical care is to achieve health. The World Health Organisation constitution defines this in terms of complete physical, mental and social well-being. For individuals with existing disorders, this entails the restoration of physical, mental and social well-being, using treatment, care and rehabilitation. In the context of chronic disease care, it is useful to consider proxies or metrics to determine health and well-ness. In People Living With HIV AIDS (PLWHA) on antiretroviral therapy (ART), commonly used metrics of well-ness include retention in care, adherence to ART, virologic suppression, and less often, functional ability or employment status. PLWHA who are engaged in care and adhere to medication may be regarded as achieving a service-level determined state of well-ness. Together with the difficult-tomeasure metric of adherence, HIV health services utilize viral load as the biologic proxy marker of adherence and well-ness. This remains the standard where both individual and servicelevel functions are ascertained. When the viral load is 
detectable, an assumption is made about poor adherence, and the absence of well-ness. This triggers a need for an intervention usually comprised of counseling, and possible referral to social or mental health services. Similar proxies may exist in chronic non-communicable diseases- for hypertension, the presence of non-adherence (and lack of well-ness) may be associated with uncontrolled blood pressure and early end-organ disease; in diabetes care, poor adherence is often associated with derangements of glycaemic control, often measured using the HBalc assay (see Steyn et $\mathrm{al}^{9}$ ).

\section{Adherence, mental disorders and barriers}

There is a growing body of literature linking poor adherence to mental disorders in PLWHA, and recent published guidelines recommend screening in order to improve rates of adherence and retention in care (see Thompson et al ${ }^{10}$ ). Individuals who screen positive for mental disorders are at high risk for meeting criteria for diagnosable disorders. In the presence of a diagnosed mental disorder, there is substantial evidence that both pharmacotherapy and psychotherapeutic interventions are effective in the treatment of comorbid mental disorders in PLWHA. 11;12 Therefore, improvements in mental disorders could potentially improve general health outcomes. Despite this, numerous barriers exist which impede the diagnosis and treatment of mental disorders, including societal, institutional and individual factors. ${ }^{13}$ In resourcelimited settings, structural and clinical barriers include lack of clinic time, high patient to staff numbers and inadequate mental health skills of primary care providers. ${ }^{14 ; 15}$ Our group recently explored some of the barriers to mental health care provision in providers working in primary health care settings in Cape Town. We noted that only about half of providers are able to recognize depression when symptoms are clearly presented; and only a third recognized substance abuse as a mental disorder. Psycho-social stress was reported more frequently then medical aetiologies as a possible cause of mental disorders. ${ }^{15}$ These all point to delays or an inability to diagnose and treat mental disorders effectively.

Amongst chronic disease clinics in resource-limited settings, point-of-contact or "proximal providers" (PPs) are typically over-burdened by numbers, focused on primary complaint or illness, and frequently lack the skills or confidence to manage mental disorders. A mental disorder often needs to be overt before it is recognized, and on the occasions where a patient is identified as suffering from a mental disorder, they are often referred to specialized services. While many of these can be managed by community-based mental health services, these services are usually run separately from other primary care services. Many community mental health services are burdened by providing services to individuals with severe mental disorders in the community and thus less able to address the burden of "common mental disorders" (depression and anxiety). ${ }^{16}$ Also, clinics are typically run by appointment and therefore temporally dis-located from general point-of-care; they may also be located at other sites, contributing to geographical dislocation. This only increases the barriers to accessing mental health services. In order to address the burden of disease in chronic communicable and non-communicable diseases, mental health care needs to be integrated into PP services.

\section{Lessons from HIV}

In HIV clinic settings, PLWHA receive care from four main provider groups: doctors, nurses, counselors and pharmacists. While doctors, and sometimes nurses, are trained in psychiatry and mental health care, it is usually the lay counselors to whom the provision of "psycho-social services" falls. This has been a necessary and pragmatic approach to the huge needs for ART preparation in high HIV prevalence areas. However, unfortunately it has led to the dis-integration of the physical and mental/social aspects of care. In increasing integration of mental health care, three key areas need to be addressed: targeting mental health care for those who most need it; training providers in mental health care; and providing effective and efficient tools for detecting mental disorders and treating them.

\section{Defining the target population}

While screening for mental disorders in all PLWHA enrolling onto ART has been advocated (see Thompson et al cited above), due to the numerous barriers previously mentioned it is likely not implementable on a wide scale basis. The resources of time and effort to screen and diagnose all individuals will most likely implode services. In HIV care, despite the high burden of psycho-social needs, the majority of PLWHA manage to adhere to ART and achieve virologic suppression. Cohort data vary across regions and times, but approximately 90\% of PLWHA are suppressed at 16 months in South Africa. ${ }^{17}$ In this study, HIV disease characteristics and gaps in care were associated with virologic failure. Current practice in South Africa is to perform the first viral load measurement after six months of ART. PLWHA who have a detectable viral load at this visit are identified and referred for counseling and support. They are then continued on first line ART and monitored for adherence within three months. This group may be regarded as a risk-of-failure sample, and mental health interventions should be targeted towards them. The aforesaid 90:10 ratio in HIV care allows for direction of resources towards an identified group in need. In noncommunicable diseases, similar markers of risk-of-failure may be described: for diabetes care, poor glycaemic control (or high $\mathrm{HBA}_{1 \mathrm{c}}$ levels) may be utilized as biomarkers of ill-health, and the need for intensive intervention.

\section{Defining and improving the intervention}

The content of the targeted intervention depends on the nature of the chronic disease, its treatment and the identified causes of possible treatment failure. The need for training in mental health, or "skills transfer" to PPs is essential. ${ }^{18}$ In HIV care settings, counselors who provide the first line of psycho-social assessment and care need to receive more intensive training in screening for mental disorders, appropriate referral pathways and practice, and advanced counseling skills. Current counselors receive between 30 and 45 days of training before entering the clinic environment. ${ }^{19}$ In addition to the provision of intensive periods of training (which may include certificate or diploma courses at colleges, training centres or universities), there is a need for ongoing structured supervision to ensure sustainability and fidelity to evidence based interventions. This may take the form of peer-led groups, or one-on-one 
supervision. The framework will depend on the available resources in the respective health district.

Another component of training is the increased access to professional mental health services at point-of-care sites. Counsellors can only be expected to provide clearly defined basic screening, referral and counseling. Diagnosis and treatment needs to fall to professional staff: nurses or doctors in primary care. Our investigations have shown that many of this cadre of worker do not feel confident to do so. ${ }^{15}$ One approach is to identify individual(s) within clinics or systems who have the time, capacity and inclination to provide these services: so-called "mental health champions" (MHCs). These nurses and/or doctors can be utilized as proximal primary providers, and can be trained in an ongoing way without draining the clinic of all its general resources (as would be required if all staff were included in the intervention). It is an acknowledgement of an approach where not all providers need to have the skills or time to provide all services, but that each system or sub-system must take responsibility to do so.

\section{Tools for mental health screening and intervention}

Following training of providers, mental health care integration and delivery may be further enhanced by the provision of tools. A "mental health toolbox" might include screening instruments, information regarding referral pathways and practice and manuals for evidence based mental health treatments. Numerous screening tools for mental disorders and psychological distress exist, and many have been validated in resource-limited settings. Screening tools need to meet the needs of the clinic population and providers it serves. In HIV clinic settings, we identified the need for a brief tool (administered in less than 10 minutes); one that identifies the mental disorders commonly associated with the physical disease (which may span only depression, anxiety and substance abuse, or require assessment of posttraumatic stress and mania in addition); and may be used by lay personnel (or counselors). We have investigated the Substance Abuse and Mental Illness Symptom Screener (SAMISS), and found it to be a potentially useful tool in HIV settings. ${ }^{20}$ In particular, counselors are able to administer the tool with acceptable reliability when compared to mental health nurses. ${ }^{21}$ In clinics providing care to other chronic diseases, suitable screeners need to be identified and their reliability and feasibility established.

\section{A model of integrated care}

A model of integrated mental health care in an HIV clinic setting is provided in figure 1. An important consideration in applying any model is that while there are health policies and systems, these are applied differently across regions and even within health districts. This means that model needs to be implemented in a way that is feasible and acceptable to the providers and managers of the respective facility. In the HIV clinic at Tier \# 1, a range of mental health skills or services may be ascribed to the respective providers, depending on their job description, level of skills and confidence. In any one clinic, a different type of provider or providers may offer these services. The clinic needs to target active screening towards individuals who are identified as being at risk-of-failure. These may amount to approximately

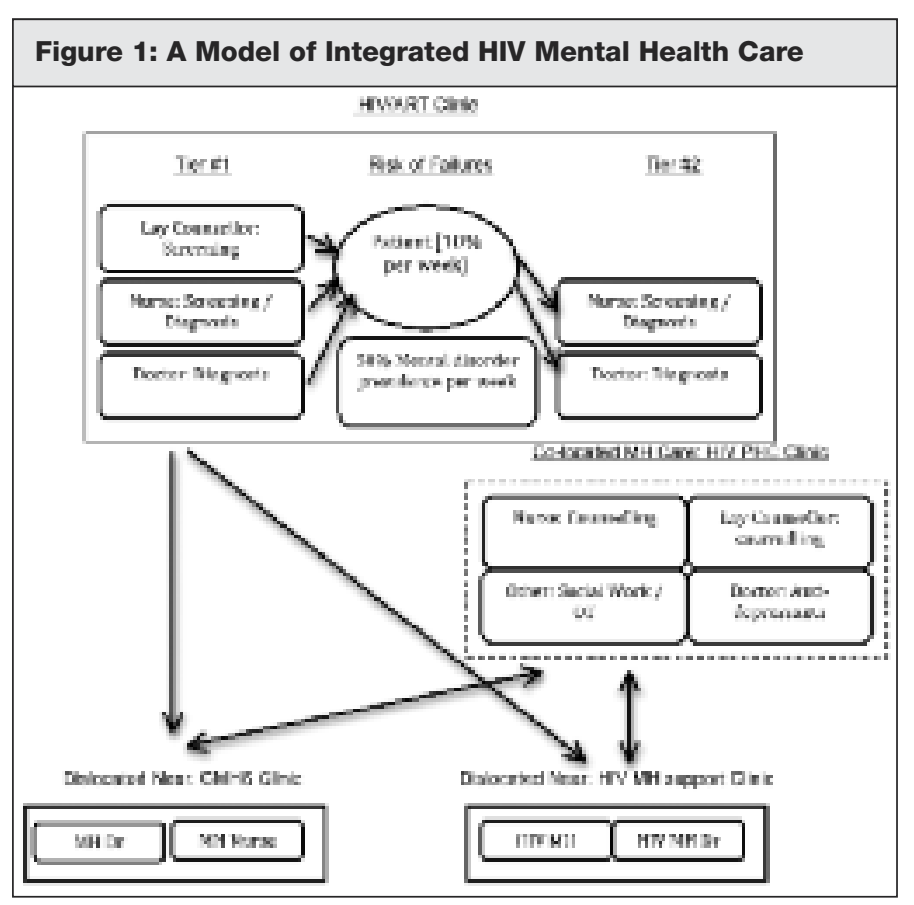

$10 \%$ of the weekly case-load. In our experience, approximately 50\% may be identified as having a mental disorder. Should the respective clinic be offering counselorlevel screening to this group, then patients will require Tier \#2: the MHC nurse or doctor needs to establish formal diagnosis, and initiate first-line treatment.

Treatment and complex assessment may be required. This bridges to the ideal of the Co-located MH Care PHC Clinic, where doctors are equipped and confident to use a range of first-line psychotropic treatments; nurses and counselors identified as MHCs will be able to offer short term psychotherapy or counseling, and social interventions can be offered by social development workers. These services assume that the Co-located MH Care PHC clinic providers are trained ("capacitated") to offer these services. In areas where such capacity is not available, then referral to dis-located services may be required- as indicated by the broken arrows in figure 1. Community mental health services (CMHS) are available by appointment, and services provided by dedicated mental health nurses and visiting medical officers or community psychiatrists. Additional resources for secondary level outreach and support in dedicated general psychiatry or neuropsychiatry services may be considered as part of a comprehensive health care plan, as annotated by the Dislocated Near HIV MH support clinic. These clinics would only see the more difficult cases. HIV MH support clinics and CMHS clinics may provide telephonic support to co-located MH Care clinics, and also refer cases down once assessment and initial management is completed.

\section{Conclusion}

Health services are undergoing rapid change with increased numbers of PLWHA on ART, and increased numbers of individuals with chronic diseases. The impact of mental disorders on long-term outcomes cannot be ignored. Mechanisms to address the mental health gap can be sought and rolled out, but they will require substantial planning and resources. Lessons from HIV care and mental health services 
suggest that an opportunity to begin to address these needs may follow a directed approach in the first instance. This way, (the smaller number of) individuals in critical need of support are helped rapidly and proximal to their place of residence. Once this is in place, other opportunities to expand mental health care exist.

\section{Acknowledgements}

We wish to acknowledge the work of our colleagues Dr Lena Andersen from the GSH-HIV Mental Health Group, Division of Neuropsychiatry, U.C.T. and Ms Lynne Wilkinson from Medicins Sans Frontieres. Working with them over the years have encouraged us to think through the issues described in this manuscript and have ultimately contributed to the development of this model.

\section{References}

1. Hock RS, Or F, Kolappa K, Burkey MD, Surkan PJ, Eaton WW. A new resolution for global mental health. Lancet 2012 ; 379(9824):1367-8.

2. Lancet Global Mental Health Group, Chisholm D, Flisher AJ, Lund C, Patel V, Saxena S, et al. Scale up services for mental disorders: a call for action. Lancet 2007 ; 370(9594):1241-52.

3. Ciesla J, Roberts J. Meta-Analysis of the Relationship Between HIV Infection and Risk for Depressive Disorders. American Journal of Psychiatry 2001;158:725-30

4. Meade CS, Sikkema KJ. HIV risk behavior among adults with severe mental illness: A systematic review. Clinical Psychology Review $2005 ; 25(4): 433-57$.

5. Fairfield K, Libman H, Davis R, Eisenberg D, Phillips R. Delays in Protease Inhibitor Use in Clinical Practice. J Gen Intern Med 1999; 14(7): 395-401

6. Starace F, Ammassari A, Trotta MP, Murri R, De Longis P, Izzo C, et al. Depression is a risk factor for suboptimal adherence to highly active antiretroviral therapy. JAIDS Journal of Acquired Immune Deficiency Syndromes 2002 ;31 Suppl 3:S136-9.

7. Ickovics JR, Hamburger ME, Vlahov D, Schoenbaum EE, Schuman P, Boland RJ, et al. Mortality, CD4 cell count decline, and depressive symptoms among HIV-seropositive women: longitudinal analysis from the HIV Epidemiology Research Study. JAMA. 2001 ;285(11):1466-74.

8. Prince M, Patel V, Saxena S, Maj M, Maselko J, Phillips MR, et al. No health without mental health. Lancet. 2007 ;370(9590):859-77.

9. Steyn K, Levitt NS, Patel M, Fourie J, Gwebushe N, Lombard C, et al. Hypertension and diabetes: poor care for patients at community health centres. S Afr Med J 2008 ;98(8):618-22.

10. Thompson M, Mugavero M, Amico R, CARgill V, Chang L, Gross R, et al. Guidelines for Improving Entry Into and Retention in Care and Antiretroviral Adherence for Persons With HIV: Evidence-
Based Recommendations From an International Association of Physicians in AIDS Care Panel [Internet]. 2012 [cited 2012 May 4]. p. 1-23. Available from: http://eutils.ncbi.nlm . nih.gov/entrez/eutils/elink.

fcgi? dbfrom=pubmed\&id=22393036\&retmode $=$ ref\&cmd = prlinks

11. Himelhoch S, Medoff DR. Efficacy of antidepressant medication among HIV-positive individuals with depression: a systematic review and meta-analysis. AIDS Patient Care STDS $2005 ; 19$ (12):813-22

12. Ferrando SJ, Freyberg Z. Treatment of depression in HIV positive individuals: A critical review. Int Rev Psychiatry 2008 ;20(1):61-71.

13. Collins PY, Holman AR, Freeman MC, Patel V. What is the relevance of mental health to HIVIAIDS care and treatment programs in developing countries? A systematic review. AIDS 2006 ;20(12):1571-82.

14. Kagee A, Delport T. Barriers to adherence to antiretroviral treatment: the perspectives of patient advocates.J Health Psychol $2010 ; 15(7): 1001-11$

15. Mall S, Sorsdahl K, Swartz L, Joska J. "I understand just a little. .". Perspectives of HIVIAIDS service providers in South Africa of providing mental health care for people living with HIVIAIDS. AIDS Care 2012;24(3):319-23.

16. Petersen I, Lund C. Mental health service delivery in South Africa from 2000 to 2010: one step forward, one step back. S Afr Med J $2011 ; 101(10): 751-7$

17. Fox MP, van Cutsem G, Giddy J, Maskew M, Keiser O, Prozesky H, et al. Rates and Predictors of Failure of First-line Antiretroviral Therapy and Switch to Second-line ART in South Africa.J Acquir Immune Defic Syndr 2012 :60(4): 428-37.

18. Petersen I, Lund C, Bhana A, Flisher AJ, Mental Health and Poverty Research Programme Consortium. A task shifting approach to primary mental health care for adults in South Africa: human resource requirements and costs for rural settings. Health Policy Plan $2012 ; 27(1): 42-51$

19. Dewing S, Mathews C, Schaay N, Cloete A, Louw J, Simbayi L. "It's Important to Take Your Medication Everyday Okay?" An Evaluation of Counselling by Lay Counsellors for ARV Adherence Support in the Western Cape, South Africa. AIDS Behav 2012 May 19; Epub ahead of print.

20. Pence BW, Gaynes BN, Whetten K, Eron JJ, Ryder RW, Miller WC. Validation of a brief screening instrument for substance abuse and mental illness in HIV-positive patients. JAIDS Journal of Acquired Immune Deficiency Syndromes 2005 ;40(4):434-44.

21. Breuer E, Stoloff K, Myer L, Seedat S, Stein DJ, Joska J. Reliability of the Lay Adherence Counsellor Administered Substance Abuse and Mental Illness Symptoms Screener (SAMISS) and the International HIV Dementia Scale (IHDS) in a Primary care HIV Clinic in Cape Town, South Africa. AIDS Behav 2012; 16(6): 1464-71. 